\title{
SUTURA DE B-LYNCH: EXPERIENCIA EN EL DESARROLLO DE UNA NUEVA TÉCNICA QUIRÚRGICA
}

\author{
Javier Troncoso M. ${ }^{1}$, Erasmo Bravo $0 .{ }^{1}$, Harald Riesle P. ${ }^{1}$, Joaquín Hevia M. ${ }^{a}$ \\ 1 Servicio de Obstetricia y Ginecología, Hospital Carlos Van Buren, Universidad de Valparaíso.
}

a Alumno, Facultad de Medicina, Universidad del Desarrollo.

\section{RESUMEN}

Antecedentes: La hemorragia postparto (HPP) continúa siendo una de las patologías más prevalentes en la morbimortalidad materna en todo el mundo, especialmente en los países subdesarrollados. Distintos protocolos de manejo y técnicas se han desarrollado en los últimos años para su control. La sutura de BLynch surge como una herramienta útil y reproducible. Objetivo: Demostrar los beneficios de la técnica para el control de la HPP. Método: Serie de 5 casos en los que se realizó la sutura de B-Lynch en el Servicio de Obstetricia y Ginecología del Hospital Carlos Van Buren. Se identifican 5 casos en los que se realizó la técnica. Resultados: En los 5 casos se logró exitosamente controlar la HPP y preservar el útero. Una de las pacientes logró un embarazo posterior exitoso. Conclusión: Nuestros resultados permiten afirmar que la técnica de B-Lynch es segura, útil y reproducible en el manejo de la HPP.

\section{PALABRAS CLAVE: sutura B-Lynch, hemorragia posparto, inercia uterina}

\section{SUMMARY}

Background: The postpartum haemorrhage (PPH) continues to be a prevalent problem affecting maternal morbimortality all over the world, particularly in non-developed countries. Different protocols of management and new techniques have been developed through these last years to control and treat PPH. The B-Lynch suture appears to be a useful and reproductible tool. Objectives: To show the benefits of the technique in the management of PPH. Method: A retrospective review of five cases in which B-Lynch suture was made, with the postoperatory controls and histeroscopics evaluations. Results: In all cases, successfully PPH was controlled and the uterus preserved. In one patient a successfully pregnancy and delivery was achieved. Conclusion: The B-Lynch suture is secure, useful and reproducible technique in the management of PPH.

\section{KEY WORDS: B-Lynch suture, postpartum hemorrhage, uterine atony}

\section{INTRODUCCIÓN}

La hemorragia postparto (HPP), definida como la pérdida de más de $500 \mathrm{~mL}$ de sangre durante las 24 horas posparto (hemorragia posparto primaria) y hasta las 12 semanas posparto (hemorragia posparto secundaria), se mantiene como una de las primeras causas de mortalidad materna en el mundo, especialmente en los países subdesarrollados. Según las estadísticas de la OMS publicadas recientemente por Khan y cols (1), el 34\% de las muertes maternas en África se deben a esta causa y el $31 \%$ en Asia. En Latinoamérica constituye la segunda causa de mortalidad con el $20,8 \%$ tras 
la patología hipertensiva (1). La realidad de Chile, es más parecida a la de los países desarrollados, representando la HPP un $6,1 \%$ en el 2000 y $3,3 \%$ en 2003 del total de la mortalidad materna de esos años (2).

La hemorragia postparto se produce principalmente por inercia uterina, persistencia de restos ovulares, lesiones del canal, retención placentaria, trastornos de la coagulación o por rotura uterina (3). No obstante la inercia uterina es la causa más prevalente y puede ser catastrófica.

La reducción de la hemorragia posparto quedó establecida en la colaboración Cochrane mediante el manejo activo del parto (4), el cual también forma parte de las recomendaciones de la OMS para el manejo del parto en todo el mundo (5). El manejo farmacológico propuesto incluye el uso de oxitocina y ergometrina. Asimismo el rol de los análogos de las prostaglandinas (misoprostol) en la profilaxis sigue estando en debate, ya que no han logrado demostrar mejores resultados que la oxitocina más ergometrina.

Frente a la persistencia del sangrado posparto, se han establecido protocolos de manejo con uso de oxitocina, ergometrina y prostaglandínicos. Pero cuando ésta persiste, se han descrito distintos procedimientos y técnicas quirúrgicas incluyendo como última medida la histerectomía obstétrica. Dentro de estas medidas está el taponamiento con balón uterino, la devascularización pelviana, la embolización arterial radiológica y suturas uterinas, y específicamente dentro de estas, está la descrita por Christopher B-Lynch, bautizada homónimamente. Una revisión reciente no encontró diferencias significativas entre estos 4 procedimientos en el control de la hemorragia posparto, con un $91,7 \%$ de éxito por parte de la técnica B-Lynch (6).

La técnica fue publicada el año 1997 con el primer reporte de 5 casos con resultados favorables, siendo el primer caso efectuado por ellos en 1989. Tras esta publicación, numerosos autores han expuesto su experiencia. En la revisión antes mencionada de Doumoutchsis y cols (6), se revisaron los resultados expuestos en 10 trabajos, con un total de 108 casos. Es así que con los casos presentados por Allahdin y cols (7), Ferguson y cols (8), Dacus y cols (9), Mazhar y cols $n(10)$ y Wergeland y cols (11), el número total de casos publicados subiría al menos en 46 más. Según reportes personales del autor de la técnica, tendría conocimiento de más de 1300 casos realizados en todo el mundo, con escasas complicaciones.

El objetivo de esta comunicación es informar el resultado de 5 casos de hemorragia posparto, tratados con la técnica de suturas de B-Lynch en el
Servicio de Obstetricia y Ginecología del Hospital Carlos Van Buren.

\section{PACIENTES Y MÉTODO}

Presentamos una serie de 5 casos en los que se realizó la técnica de sutura de B-Lynch para el control de la hemorragia posparto, con control histeroscópico tardío de la condición de la cavidad uterina, en aquellas pacientes que fue factible de realizar. Las pacientes fueron atendidas en el Servicio de Obstetricia y Ginecología del Hospital Carlos Van Buren, en el período comprendido entre febrero de 2005 a febrero de 2007.

Descripción de la técnica:

1.- Paciente en posición de Lloyd Davies, que permite acceso vaginal y clara visualización del sangrado por esa vía. El segundo ayudante debe situarse en esa posición.

2.- Una vez en la cavidad abdominal, se realiza histerotomía segmentaria, previo rechazo de la vejiga, o en caso de cesárea reciente se abren los puntos de la histerorrafia. La cavidad uterina es examinada y evacuada de todo resto o coágulo que persista en su interior.

3.- El útero debe ser exteriorizado y debe comprimirse con ambas manos por el ayudante (una por la cara anterior del útero y otra por la cara posterior), de cesar el sangrado se presume una buena respuesta a la técnica.

4.- $\mathrm{Si}$ el sangrado se detuvo, el cirujano debe iniciar el procedimiento con una aguja semicircular roma de $70 \mathrm{~mm}$, montada con sutura monocryl №1, tal como se ve en la Figura 1 y 2 . El primer punto es puesto $3 \mathrm{~cm}$ bajo la histerotomía, entrando a la cavidad y emergiendo $3 \mathrm{~cm}$ por sobre el borde superior, aproximadamente a $4 \mathrm{~cm}$ del borde lateral del útero. Luego, el material de sutura es llevado por sobre el útero hacia la cara posterior evitando que se deslice por el borde del ligamento ancho. En la cara posterior, a nivel de la histerotomía, se introduce nuevamente en la cavidad uterina, para luego emerger nuevamente en la cara posterior. Hecho esto, nuevamente se lleva el material por sobre el útero en el lado contralateral hacia la cara anterior, donde nuevamente se ingresa a la cavidad a $3 \mathrm{~cm}$ por sobre el borde superior de la histerotomía, saliendo a $3 \mathrm{~cm}$ bajo la histerotomía, a $4 \mathrm{~cm}$ del borde lateral del útero. Durante todo el procedimiento el ayudante debe mantener el útero comprimido, ya que de esta forma disminuye el riesgo de deslizamiento del material de sutura por el ligamento ancho y evita el trauma uterino.

5.- Ambos cabos deben mantenerse tensos y se pueden anudar con doble nudo, antes o después 
de realizar la histerorrafia, con la salvedad de que si se anuda antes, es recomendable primero realizar una sutura de ángulo a cada lado, porque tiende a ser más difícil posteriormente.

La técnica original fue publicada utilizando catgut cromado №2 de $75 \mathrm{~cm}$ y aguja triangular. La revisión de otras publicaciones comunica el uso de otros materiales de sutura como dexon, PDS, prolene y nylon. Particularmente en nuestros casos hemos usado vycril №1. Acorde a la última recomendación del autor (12), la sutura ideal sería un monofilamento reabsorbible, como es el monocryl, con un largo adecuado (idealmente $90 \mathrm{~cm}$ ) y con aguja suficientemente grande y cómoda.

Existen distintas características entre los materiales de sutura, y según la recomendación del autor, el monocryl es la ideal por su rápida absorción, que impediría la producción de lesiones en órganos vecinos, en aquellos casos que la sutura se soltara

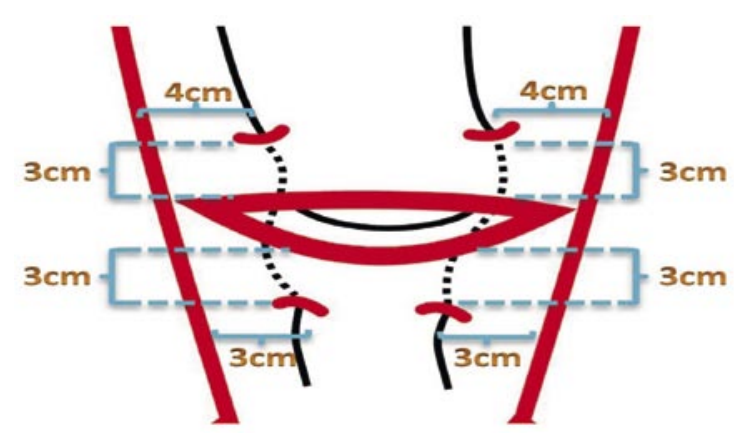

Figura 1. Sutura de B-Lynch: puntos de entrada y salida en relación a la histerotomía. y deslizara por el ligamento ancho, una vez que el útero vuelva a su tamaño normal.

\section{RESULTADOS}

Caso 1. Paciente de 22 años, G3POA2 (abortos espontáneos a las 10 y 12 semanas), sin antecedentes mórbidos de importancia. Ingresa en trabajo de parto a las $40+1$ semanas. Parto vaginal espontáneo sin complicaciones a las 5 horas de su ingreso, bajo anestesia de conducción. Propagación de la episiotomía, que se repara inmediatamente. Recién nacido vivo de 3660 gramos. A las 2 horas posparto la paciente inicia sangrado vaginal importante, taquicardia e hipotensión arterial, respondiendo satisfactoriamente a las medidas iniciales. Pasadas 15 horas posparto, nuevamente presenta metrorragia moderada, palidez, con ciclo vital estable. Es llevada a pabellón, realizándose revisión instrumental con salida de coágulos, sin evidencia de daño del canal del parto y escasa respuesta a las medidas terapéuticas. Se decide intervención quirúrgica de B-Lynch, que se efectúa sin complicaciones. La paciente tiene una buena respuesta postoperatoria, con estabilización hemodinámica, recibió $4 U$ de glóbulos rojos por caída de hematocrito a $14 \%$. Alta al 4 día. El control histeroscópico al 4 mes visualiza sinequia corporal lateral izquierda que se debrida fácilmente, persistiendo sólo el solevantamiento de fondo uterino (tipo arcuato). La paciente reingresa 11 meses después del procedimiento, con diagnóstico de aborto incompleto de 12 semanas. Se realizó legrado uterino sin complicaciones.

Caso 2. Primigesta, 17 años, embarazo de $39+3$ semanas, ingresa para cesárea electiva con los

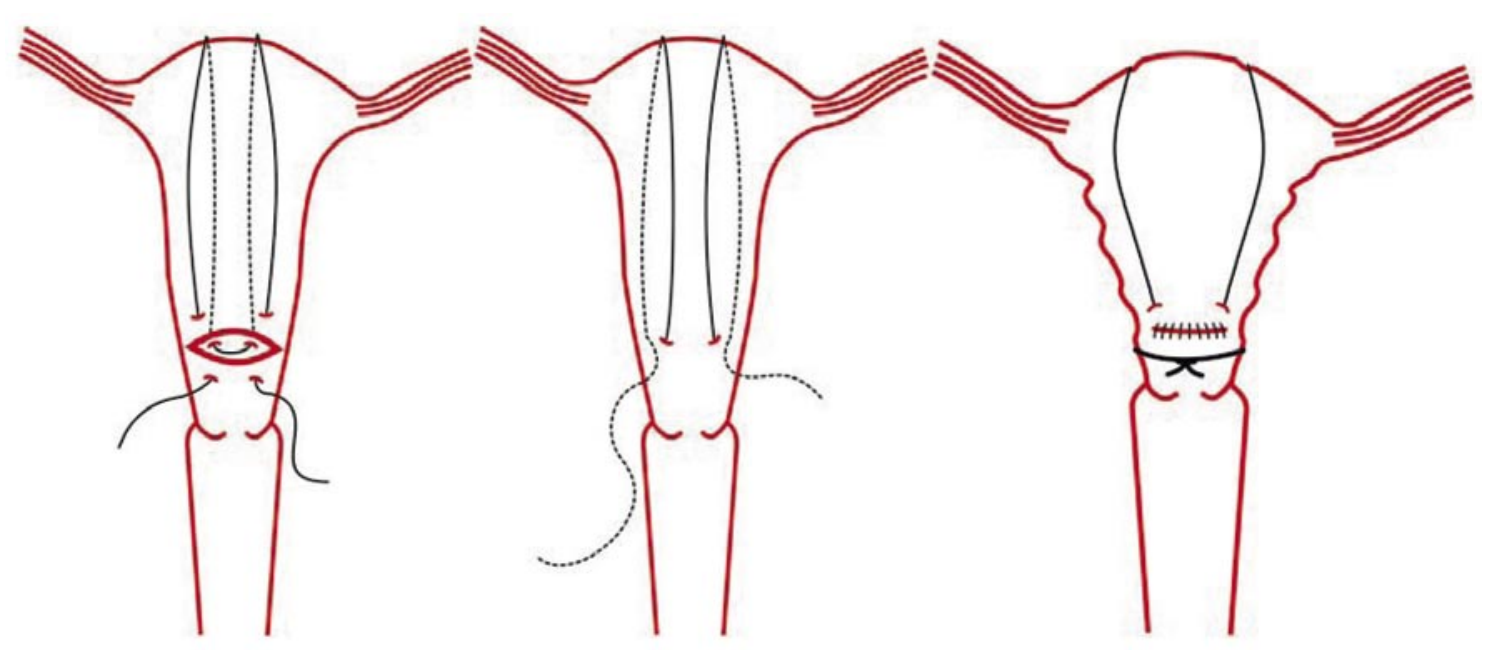

Figura 2. Vista anterior y posterior uterina mostrando la sutura de B-Lynch. 
diagnósticos de macrosomía fetal y oligoamnios. Recién nacido de 4.500 gramos. Durante la cesárea se comprueba inercia uterina al término de la histerorrafia. Se procede al manejo farmacológico habitual y masaje uterino. Por persistencia del sangrado se procede a abrir la histerorrafia y explorar la cavidad uterina, decidiéndose sutura de B-Lynch. Se completa el cierre de la laparotomía. La paciente tiene buena respuesta postoperatoria, con anemia severa posparto que no requirió transfusión sanguínea. Histeroscopia de control a los 7 meses es informada como cavidad uterina indemne.

Caso 3. Paciente de 33 años de edad multípara de dos partos vaginales, el último hace 8 años. Ingresa cursando 25 semanas de embarazo derivada desde el hospital Claudio Vicuña de San Antonio, con diagnóstico de óbito fetal por desprendimiento prematuro de placenta normoinserta, sin respuesta a la inducción con misoprostol y coagulopatía de consumo secundaria. Ingresa febril ( $38^{\circ} \mathrm{C}$ axilar), taquicárdica, normotensa, pálida y sudorosa. Al examen segmentario destaca abdomen blando, con útero hipertónico de $30 \mathrm{~cm}$ doloroso a la palpación. Los exámenes de ingreso muestran hematocrito de $23,7 \%$ y plaquetopenia, con pruebas de coagulación alteradas (TP 68,9\% y TTPK > 180 segundos). Se indica cesárea de urgencia que evidencia útero de Couvelaire. Se comprueba desprendimiento de placenta masivo con gran hematoma retroplacentario (1 litro aproximadamente) e infiltración miometrial hemática severa. Evoluciona de inmediato con inercia uterina, procediéndose a colocar sutura de B-Lynch. Se comprueba cese del sangrado uterino y se concluye la cirugía preservando el útero. Para su estabilización hemodinámica y corrección de la coagulopatía requirió 6 unidades de plaquetas, 6 unidades de plasma fresco congelado y 3 unidades de glóbulos rojos. Alta en buenas condiciones. Histeroscopia de control a los 90 días muestra cavidad uterina normal y sin sinequias.

Caso 4. Paciente de 31 años, con cicatriz de cesárea anterior por parto prematuro extremo y distocia de presentación. Ingresa cursando embarazo de 36 semanas y rotura prematura de membranas de 12 horas de evolución. Al completar 24 horas e iniciado tratamiento antibiótico se decide cesárea. Recién nacido de 3.550 gramos. Durante la cirugía se visualizan múltiples adherencias y acretismo placentario en pared anterior en relación a cicatriz previa. Tras la extracción fetal y el alumbramiento dirigido, se pesquisa atonía uterina y persistencia del sangrado en pared anterior, decidiéndose sutura B-Lynch, sin complicaciones. La paciente se traslada fuera del país no pudiéndose realizar control histeroscópico tardío.
Caso 5. Paciente de 30 años, G2P0A1 (aborto retenido de 12 semanas con legrado uterino 2 años antes). Ingresa para cesárea electiva por macrosomía fetal, cursando embarazo de término de 39+4 semanas. Recién nacido de 4.220 gramos. Durante la cesárea se pesquisa inercia uterina, con escasa respuesta al tratamiento habitual (oxitocina + ergometrina + misoprostol). Se decide sutura de BLynch con prolene. Alta en buenas condiciones al tercer día. Por motivos de la paciente no fue posible control histeroscópico tardío. Un año después embaraza espontáneamente, resolviéndose por cesárea electiva debido a sus antecedentes. Durante la cesárea se encuentra como hallazgo la presencia de una gruesa banda entre la pared anterior uterina y la pared abdominal, que se libera sin complicaciones. Recién nacido de 3.850 gramos. Postoperatorio sin complicaciones.

\section{DISCUSIÓN}

Además de los casos expuestos, el número de histerectomías obstétricas realizadas en el Servicio durante los últimos años, ha tenido un descenso sostenido hasta 2006 (Figura 3), teniendo dentro de las causas por las cuales se realizó la cirugía a la inercia uterina en un $85,1 \%(40 / 47)$ y al acretismo placentario en un $14,9 \%(7 / 47)$.

Tal como se demuestra en los casos expuestos, la técnica probó ser una solución eficiente, útil, rápida y fácilmente reproducible, y que fue utilizada para distintas causas de hemorragia posparto, no sólo por inercia uterina, sino también por cuadros derivados de desprendimiento prematuro de placenta norminserta y acretismo placentario.

Distintas revisiones del tema han logrado establecer que durante la última década se han desarrollado nuevas técnicas para el control de la HPP (13). Sin embargo, algunas requieren de una experticia técnica alta como la devascularización pelviana

\section{Histerectomías Obstétricas}

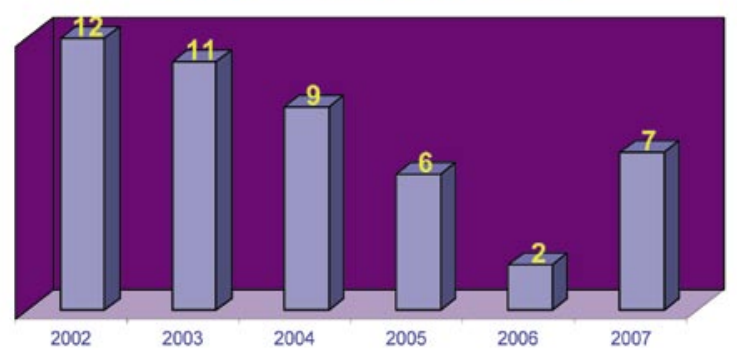

Figura 3. Número de histerectomías obstétricas realizadas en el período 2002-2007. 
(14) o de una alta complejidad en los servicios de apoyo, como en la embolización arterial radiológica (15), además de requerir una estabilidad hemodinámica difícil de lograr en pacientes con sangrado activo. Ante esta situación, la sutura de B-Lynch se plantea como una opción segura y simple.

Dentro de las complicaciones vistas en las pacientes, sólo se encontró en una paciente una sinequia uterina laxa, que fue debridada sin complicaciones, logrando aunque sin éxito, un nuevo embarazo posterior. En otra paciente se pudo observar la presencia de una gran banda fibrosa, que no impidió ni complicó el embarazo posterior, posiblemente debido al uso de material no reabsorbible en el procedimiento.

Se han descrito variaciones de la técnica original, como las establecidas por Hayman y cols (16), Cho y cols (17) y Bhal y cols (18), como se aprecia en las Figuras 4, 5 y 6 , que buscan perfeccionar la técnica original y disminuir los tiempos operatorios. Se han reportado complicaciones postoperatorias como la necrosis uterina (19) y piometra postoperatoria (20), que coincidentemente se han relacionado con las variaciones de la técnica, ya que no cumplen con ciertos principios que tiene la técnica original, como es la exploración de la cavidad uterina, limpiándola de coágulos y restos ovulares y la garantía de un vaciamiento uterino normal por el orificio cervical interno, al no transfixiar la cavidad uterina, evitando así, la acumulación de material intrauterino. Es por esto que en los casos en que hemos decidido ocupar la técnica, se ha preferido el reporte original.

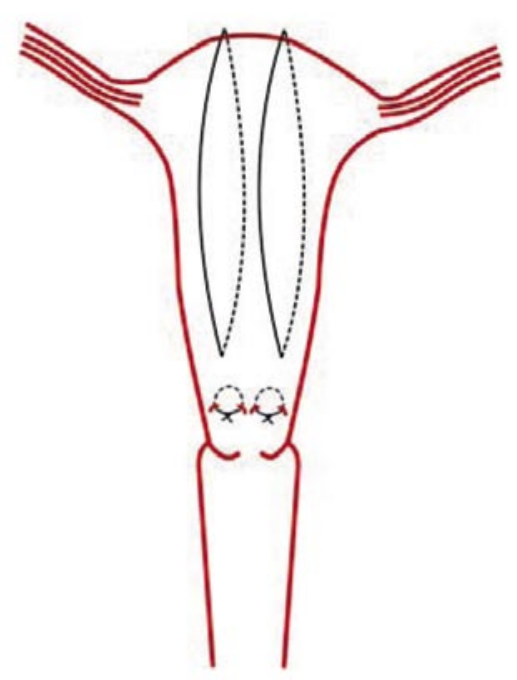

Figura 4. Modificación de Hayman y cols (referencia 16).

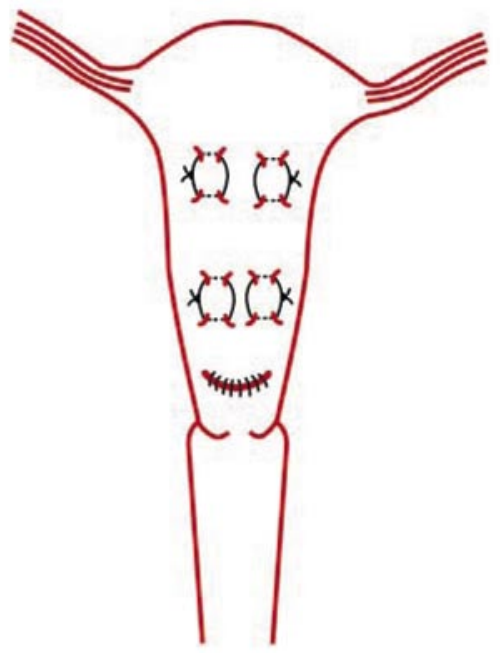

Figura 5. Modificación de Cho y cols (referencia 17).

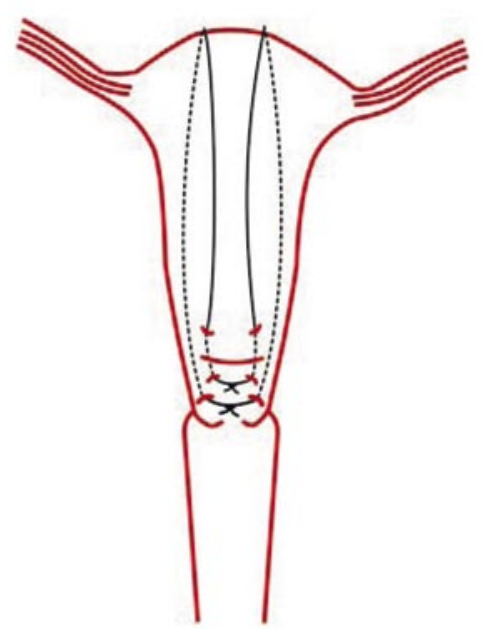

Figura 6. Modificación de Bhal y cols (referencia 18).

La hemorragia postparto persiste siendo un problema de salud en el mundo y en nuestro país, por lo tanto, todos los esfuerzos dirigidos hacia la prevención y su tratamiento, va a tener como consecuencia un resultado positivo en la disminución de la morbimortalidad materna. La técnica desarrollada por Christopher B-Lynch permitió en nuestros casos una resolución exitosa de situaciones clínicas complejas, solucionando la hemorragia posparto conservando el útero, permitiendo al menos que una de las pacientes pudiera concretar un embarazo posterior exitoso. 


\section{CONCLUSIÓN}

Se comunica la experiencia de 5 casos de control del sangrado severo posparto aplicando la técnica de sutura de B-Lyinch, que permitió evitar la histerectomía obstétrica y la conservación de la capacidad reproductiva en al menos 2 pacientes. La técnica quirúrgica demostró ser segura, reproducible y fácil de realizar por especialistas en obstetricia y ginecología, por lo que se recomienda su incorporación dentro de las técnicas quirúrgicas para el control de la hemorragia posparto.

\section{BIBLIOGRAFÍA}

1. Khan KS, Wojdyla D, Say L, Gülmezoglu AM, Van Look PF. WHO analysis of causes of maternal death: a systematic review. Lancet 2006; 367(9516):106674.

2. Donoso E, Oyarzún E. Mortalidad materna, Chile 2003: ¿Continúa el gran descenso? Rev Chil Obstet Ginecol 2005;70(2):79-82.

3. Anulkumaran $\mathrm{S}$, de Cruze B. Surgical management of severe postpartum hemorrhage. Curr Obstet Gynaecol 1999;9:101-5.

4. Prendiville WJ, Elbourne D, McDonald SJ. Active versus expectant management in the third stage of labor. Cochrane Database Syst Rev 2009;(3):CD000007. Review.

5. WHO recommendations for prevention of postpartum haemorrhage. 2000. Hallado en: http://www.who.int/ making_pregnancy_safer/publications/WHORecommendationsforPPHaemorrhage.pdf

6. Doumouchtsis S; Papageorghiou A; Arulkumaran S. Systematic review of conservative management of postpartum hemorrhage: what to do when medical treatment fails. Obstet Gyn Surv 2007;62(8):540-7.

7. Allahdin S, Aird C, Danielian P. B-Lynch sutures for major primary postpartum haemorrhage at caesarean section. J Obstet Gynaecol 2006;26(7):639-42.

8. Ferguson JE, Bourgeois FJ, Underwood PB. B-Lynch suture for postpartum hemorrhage. Obstet Gynecol 2000;95:1020-2.

9. Dacus JV, Busowski MT, Busowski JD, Smithson S, Masters K, Sibai BM. Surgical treatment of uterine atony employing the B-Lynch technique. J Matern Fetal Med 2000;9(3):194-6.

10. Mazhar S. Management of massive postpartum hemorrhage by B-Lynch brace suture. J Coll Phys Surg Pak 2003;13: 51-2.

11. Wergeland $\mathrm{H}$. Use of the B-Lynch suture technique in postpartum hemorrhage. Tidsskr Nor Laegeforen 2002;122:370-2.

12. Price N, B-Lynch C. Technical description of the BLynch brace suture for treatment of massive postpartum hemorrhage and review of published cases. Int $\mathrm{J}$ Fertil Womens Med 2005;50(4):148-63.

13. Rebarber A, Roman A. Cover Story: seven ways to control postpartum hemorrhage. Contemporary Obtet Gyncol 2003;3:34-53.

14. O'Leary JA. Uterine artery ligation in the control of postcesarean hemorrhage. J Reprod Med 1995;40:18993.

15. Royal College of Obstetricians and Gynaecologist. The role of emergency and elective interventional radiology in postpartum haemorrhage. Good Practice No 6. June 2007.

16. Hayman RG, Arulkumaran S, Sreer PJ: Uterine compression sutures: surgical management of postpartum haemorrhage. Obstet Gynecol 2002;99(3):502-6.

17. Cho JH, Jun HS, Lee CN. Haemostatic suturing technique for uterine bleeding during cesarean delivery. Obstet Gynecol 2000;96:129-31.

18. Bhal K, Bhal N, Mulik V, Shankar L. The Uterine compression suture -- a valuable approach to control major postpartum haemorrhage at lower segment cesarean section. J Obstet Gynaecol 2005;25(1):10-4.

19. Treloar EJ, Anderson RS, Andrews HS, Bailey JL. Uterine necrosis following B-Lynch suture for primary postpartum haemorrhage. BJOG 2006;113:486-8.

20. Ochoa M, Allaire AD, Stitely ML. Pyometria after haemostatic square suture technique. Obstet Gynecol 2002;99:506-9. 